\title{
PERFIL DE MEDICAMENTOS UTILIZADOS VIA SONDA ENTERAL EM PEDIATRIA
}

\section{DRUG PROFILE UTILIZED IN ENTERAL FEEDING TUBE IN PEDIATRICS}

\author{
Harli Pasquini NETTO ${ }^{1 *}$, Caroline Kaori MAEBAYASHI ${ }^{2}$, Isabela Maria Coatti \\ ROCHA ${ }^{2}$, Denise Tiemi MIYAKAWA ${ }^{3}$, Mirella Aparecida NEVES ${ }^{4}$, Jocemara \\ GURMINI $^{5}$, Talita Bux ABAGE ${ }^{6}$, Rosiane Guetter Mello ZIBETTI ${ }^{7}$
}

1 - Farmacêutico, Especialista em Saúde da Criança e do Adolescente pelas Faculdades Pequeno Príncipe, mestrando no Programa de Pós-graduação em Ciências Farmacêuticas da Universidade Federal do Paraná.

2 - Discente do curso de Medicina das Faculdades Pequeno Príncipe, Curitiba - PR.

3 - Médica Gastroenterologista e Nutrologista Pediatra. Mestranda em Biotecnologia Aplicada à Saúde da Criança e do Adolescente pelas Faculdades Pequeno Príncipe, Curitiba - PR.

4 - Nutricionista Clínica. Mestranda em Biotecnologia Aplicada à Saúde da Criança e do Adolescente pelas Faculdades Pequeno Príncipe, Curitiba - PR.

5 - Doutoranda em Biotecnologia Aplicada à Saúde da Criança e do Adolescente pelas Faculdades Pequeno Príncipe, Curitiba - PR.

6 - Nutricionista do Hospital Pequeno Príncipe, Curitiba - PR.

7 - Farmacêutica. Mestre e Doutora em Bioquímica pela Universidade Federal do Paraná.

*Autor para correspondência: harli.pasquini@ufpr.br

\section{RESUMO:}

Objetivo: O presente artigo procura avaliar o perfil da terapia medicamentosa utilizada via sonda enteral em um hospital pediátrico com o intuito de detectar possíveis inadequações terapêuticas. Com isso, acaba abordando o manuseio das sondas e a maneira correta de as utilizar, além de propor cenários preveníveis de possíveis inadequações terapêuticas. Método: Foi realizado um estudo descritivo do perfil da farmacoterapia utilizada via sonda enteral, exploratório nos meses de Maio e Junho de 2015, por uma população de caráter pediátrico. Resultados: Foi constatado que dos pacientes a média de medicamentos administrados via sonda era $5,89 \pm 4,30$, em que a maioria é utilizada off-label. Sendo do total de medicamentos prescritos, os de forma líquida apresentaram maiores discrepâncias entre a via prescrita e a via administrada. Além disso, $46,83 \%$ dos casos o flushing era efetuado apenas após a administração dos fármacos. Conclusão: Conclui-se que um acompanhamento farmacoterapêutico diário detalhado é vital para garantir uma terapia farmacológica eficaz e segura em pediatria.

Palavras-Chave: Nutrição enteral; pediatria; Uso Off-Label.

ABSTRACT:

Objetive: This paper aims on evaluating the drug therapy profile utilized through enteral feeding tubes in a pediatric hospital in order to detect potential terapeutic inadequacies. Therefore, deals with the enteral feeding tubes handling and its inadequacies, also propose 
preventable scenarios based on possible therapeutic inadequacies. Methods: It was conducted a descriptive study about the drug therapy profile utilized through enteral tubes, with a exploratory character during May and June of 2015, in a pediatric population. Results: In average, patients with a enteral feeding tube were submitted to $5.89 \pm 4.30$ different drugs, which the majority were off-label. The liquid drugs were the presentation which had more discrepancies between prescription and its real administration. Moreover flushing was done only after drug administration, corresponding to $46.83 \%$ of the cases. Conclusion: It was concluded that a daily pharmacotherapeutic follow-up is vital to ensure an effective and safe therapy in pediatrics.

Key-words: Enteral Nutrition; Pediatric; Off-Label Use.

\section{INTRODUÇÃO}

Em pacientes pediátricos é comum o uso de sonda enteral (SE), em que há administração direta no trato gastrointestinal (TGl), assim, evitando a via oral ${ }^{1}$. Um dos motivos da administração de medicamentos por via SE é justificado pela incapacidade dos pacientes se nutrirem por esta via e pela insuficiência na aquisição de nutrientes pela mesma, seja por redução da capacidade absortiva ou por disfagia ${ }^{2}$. Essa via se divide em sonda nasogástrica (SNG), sonda nasoenteral (SNE), sonda orogástrica (SOG), sonda oroenteral (SOE), gastrostomia (GT) e jejunostomia (JT) ${ }^{3}$.

Apesar da utilização off-labe/ ${ }^{4}$, esta prática é realizada rotineiramente no âmbito da pediatria, mesmo havendo escassez na literatura sobre a farmacoterapia, além de não ser comumente tão abordada pelos estudos clínicos realizados pelos laboratórios farmacêuticos. Em um levantamento realizado em São Paulo, 40,8\% dos medicamentos eram de uso off-labe/.

Nas unidades de internação, em que é utilizado tratamento por via SE, é comum ocorrerem casos de complicações por manuseio inadequado. Dentre esses, destacam-se a administração concomitante de alimento-medicamento, como exemplificado por Izco et al. ${ }^{5}$, podendo esses interagirem entre si ou entre medicamento-medicamento, devido a uma interação físico-química na qual um fármaco interage fora do organismo ${ }^{6}$, sendo isso denominado incompatibilidade ou interação farmacêutica.

Outra inadequação é a não realização frequentemente do flushing (ou lavagem) da sonda enteral (SE), o que pode causar transtornos ao paciente e gastos extras para o hospital por obstruir a sonda, necessitando de substituição da mesma. Como mostrou o estudo de Pérez-Guisado7 ${ }^{7}, 16,7 \%$ dos pacientes que tiveram que trocar a sonda nasoenteral (SNE) por uma obstrução, ocorreram devido a consequências de flushing inadequado ou ausência deste.

Portanto, todas essas complicações podem ser prevenidas pela utilização correta da 
sonda e pela investigação do perfil farmacoterapêutico. Assim, é de extrema importância descrever o perfil de utilização de medicamentos via sonda, principalmente quando se refere à pediatria, para o uso adequado da medicação. Neste sentido o presente se diferencia pela identificação de alguns fatores que comprometem 0 tratamento farmacoterapêutico.

\section{MÉTODO}

O presente estudo é de natureza quantitativa de cunho exploratório-descritivo, foi realizado por meio de uma pesquisa documental transversal, em um hospital de alta complexidade pediátrica, em Curitiba, Paraná, Brasil. Na pesquisa foi realizada uma análise dos prontuários médicos e evoluções eletrônicas da equipe de enfermagem, que apresentassem medicamentos prescritos via sonda enteral ou que utilizassem medicamentos por esta via, mesmo prescrito por outra. Os dados foram coletados entre os meses de Maio e Junho de 2015. A busca ativa de prescrições de pacientes portadores de sonda enteral,foi composta de 75 prescrições. Os dados coletados foram: dados do paciente: idade, gênero, unidade de internação, tipo de sonda que utiliza; dados da terapia: nome do princípio ativo, forma farmacêutica, diluição, vias de administração prescrita e realizada, realização de flushings. Esses dados foram avaliados sobre os aspectos de distribuição do gênero, idade dos portadores e tipo de sonda utilizada, média e desvio padrão dos medicamentos utilizados por esta via de admisnistração, o perfil da divergência da via de administração em relação à via prescrita, ocorrência de flushing no ato da administração. A análise foi realizada por meio de estatística descritiva com o auxílio do Microsoft Excel 2010.

Neste artigo, o termo líquido representa o conjunto das formas farmacêuticas xarope, solução e suspensão. O projeto foi aprovado pelo Comitê de Ética em Pesquisa em Seres Humanos sob o CAAE 44281415.5.0000.5580.

\section{RESULTADOS}

\subsection{Perfil dos pacientes portadores de sonda enteral}

Neste estudo foram avaliados 75 pacientes portadores de sonda enteral, onde cada um desses utilizou cerca de 5,89 $\pm 4,30$ medicamentos, por essa via de administração. Dos 
pacientes, $56 \%$ ( $n=42)$ são do gênero masculino e 44\% ( $n=33)$ são do gênero feminino. Do total de pacientes uma grande parte $(29,33 \%, n=22)$ possuíam idade menor que 6 meses de vida, 10,67\% $(n=8)$ de 6 a 11 meses, $16 \%(n=12)$ de 1 a 2 anos, $12 \%(n=9)$ de 3 a 5 anos, 10,67\% $(n=8)$ de 6 a 9 anos, $8 \%(n=6)$ de 10 a 13 anos, 13,33\% $(n=10)$ de 14 a 18 anos.

Também foi constatado que as sondas para terapia de curto prazo (SNE, SOE, SOG e SNG) representavam 49,33 \% $(n=37)$ dos tipos de sondas utilizadas, já as gastrostomias (GT) utilizadas em terapias prolongadas, representam $50,67 \%(n=38)^{8}$.

\subsection{Sondas utilizadas $X$ Unidades de internação}

As Unidades de Internação (UI) que apresentaram o uso gastrostomia foram: 6,67\% ( $n=8)$ na Clínica Pediátrica Geral, 6,67\% $(n=8)$ na Unidade de Tratamento Intensivo (UTI) Neonatologia, 6,67\% ( $\mathrm{n}=5)$ na UTI Pediátrica Geral, 5,33\% $(\mathrm{n}=4)$ na UI por convênio II, 4,00\% ( $n=3)$ na UI Neurologia, 4\% $(n=3)$ na UI convênio apartamento I , 4\% ( $n=3)$ na UI convênio apartamento II, 4\% $(n=3)$ na UI Cirúrgica, 2,67\% $(n=2)$ no Ambulatório Nutrologia, 2,67\% $(n=2)$ no UI Convênio Coletivo I, 1,33\% $(n=1)$ na UTI Cirúrgica, 1,33\% $(n=1)$ na UI Ortopedia, $0 \%(n=0)$ na UTI Cardiológica, $0 \%(n=0)$ na UI Cardiológica, $0 \%$ $(n=0)$ na UI Hemato-oncologia, somando um total de 50,67\% ( $n=38)$.

As sondas de terapia curta foram contabilizadas em 49,33\% ( $n=37)$ dos pacientes analisados, onde ocorreram 16\% $(n=12)$ na UTI Cardiológica, 6,67\% $(n=5)$ na UI Neurologia, 5,33\% $(n=4)$ na Clínica Pediátrica Geral, 5,33\% ( $n=4)$ na UTI Geral, 4,00\% $(n=3)$ na UI Cardiologia, 2,67\% $(n=2)$ na UTI Neonatologia, 2,67\% $(n=2)$ na UI Convênio Apartamento I, 2,67\% $(n=2)$ na UTI Cirúrgica, 1,33\% $(n=1)$ na UI Convênio Apartamento II, $1,33 \%(n=1)$ na UI Hemato-Oncologia, 1,33\% $(n=1)$ no Ambulatório Nutrologia, 0\% ( $n$ $=0)$ na UI Convênio Coletivo I, $0 \%(n=0)$ na UI Convênio Coletivo II, $0 \%(n=0)$ na UI Ortopedia, $0 \%(n=0)$ na UI Cirúrgica.

\subsection{Perfil dos medicamentos utilizados via sonda enteral}

Na Tabela 1, está o perfil dos medicamentos utilizados via sonda. Sendo que os mais utilizados foram fenobarbital (5,66\%), paracetamol (5,66\%), polivitamínico (4,07\%). Já o perfil das principais classes de medicamentos analisadas, $24,43 \%$ foram anticonvulsivantes, $11,54 \%$ foram vitaminas, analgésicos e antitérmicos foram $8,60 \%$ e $3,39 \%$ de antimicrobianos. 
Tabela 1. Perfil dos principais medicamentos e classes de medicamentos administrados via sonda.

\begin{tabular}{lcc}
\hline & Número Absoluto & Porcentagem (\%) \\
\hline Medicamentos & TOTAL $=442$ & Correspondente a 100\% \\
Fenobarbital & 25 & $5,66 \%$ \\
Paracetamol & 25 & $5,66 \%$ \\
Polivitamínico & 18 & $4,07 \%$ \\
Ácido fólico & 16 & $3,62 \%$ \\
Clobazam & 16 & $3,62 \%$ \\
Simeticona & 12 & $2,71 \%$ \\
\hline
\end{tabular}

\section{Principais classes de medicamentos utilizados via sonda}

Anticonvulsivantes

Vitaminas

Analgésico/Antitérmico

Antimicrobianos

108

51

38

15
$24,43 \%$

$11,54 \%$

$8,60 \%$

$3,39 \%$

Na tabela 1, denominada "Perfil dos principais medicamentos e classes de medicamentos administrados via sonda", observa-se os principais medicamentos administrados. Sendo que na primeira coluna da esquerda encontra-se, respectivamente, seus nome e classes. Já nas colunas seguintes, há a proporção de cada um desses em valores absolutos e, em seguida, em porcentagem.

A Figura 1 descreve o perfil das formas farmacêuticas utilizadas via sonda, onde foi obtido: $35,07 \%$ em gotas, $28,28 \%$ de líquidos, $22,62 \%$ de comprimidos, $7,92 \%$ de comprimidos já diluídos pelo Serviço de farmácia Hospitalar (SFH), 4,30\% de pó/grânulo e apenas $1,81 \%$ de cápsulas.

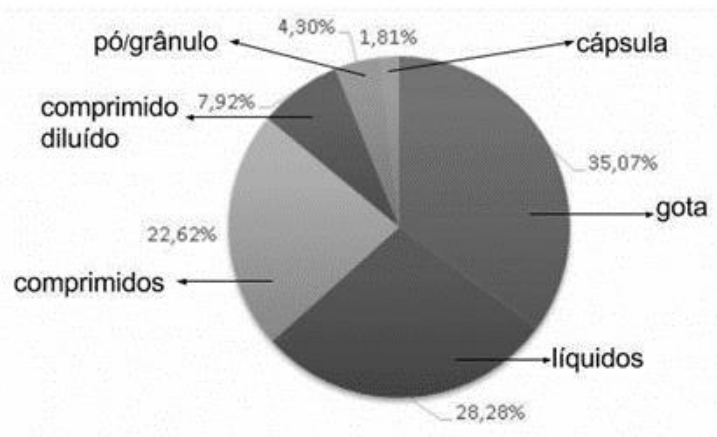

Figura 1. Perfil das formas farmacêuticas utilizadas 
Na Figura 1, de título "Perfil das formas farmacêuticas utilizadas", há análise da proporção de medicamentos utilizados conforme forma farmacêutica. Como nas sondas o ideal é utilizar formas líquidas,o presente estudo o realiza com $71,27 \%$ da proporção de medicamentos, somatória das formas gota, comprimido diluído e líquidos. Já o restante, equivalente a 28,73\%, não o alcançavam. Sendo que desse, 22,62\% correspondem a comprimidos, os quais poderiam ser diluídos ou substituídos pela forma em gotas conforme apresentado nas análises do texto.

\subsection{Perfil de divergências entre medicamentos prescritos $\mathrm{x}$ realizados}

No presente estudo, contabilizou-se 126 casos de divergências por medicamento prescrito, na qual 46,03\% ( $n=58$ ) ocorreu em sonda gástrica e 53,97\% ( $n=68)$ ocorreu em sonda enteral. Já nas formas farmacêuticas pode-se observar maior discrepância nos líquidos, com 47 inadequações das 125 formas farmacêuticas prescritas, correspondendo a $37,6 \%$. Seguido de gotas, em que de 155 , o percentual de inadequações dos $29,67 \%$ (n = 46). Em seguida, dos 100 comprimidos prescritos, 35 apresentavam inadequações (35\%). Em comparação, dos 35 comprimidos diluídos, 20 ocorreram discrepâncias (57,14\%). Por fim, quanto as inadequações de pós/ grânulos e as cápsulas notou-se $47,37 \%$ ( $n=19)$ e $12,5 \%$ (1 de um total de 8 ), respectivamente.

\subsection{Flushing}

Das 442 administrações de medicamentos, $52,26 \%(n=231)$ dos medicamentos foram realizados flushings antes e após as suas administrações, 46,83\% ( $n=207)$ realizados flushing apenas após e $0,90 \%(n=4)$ realizados flushing apenas antes do administração dos medicamentos.

\section{DISCUSSÃO}

\subsection{Perfil de pacientes portadores de sonda enteral}

Neste estudo, dos 75 pacientes portadores de sonda analisados 29,33\% possuíam idade inferior a 6 meses de idade, evento previsto nessa faixa etária pela formação completa do sistema digestório ocorrer aproximadamente no oitavo mês de vida ${ }^{9}$.

Apesar das sondas para terapia de curto prazo, como sonda SNG, SOG, SNE,SOE serem comumente mais utilizadas pela sua facilidade no manuseio e menor custo ${ }^{10}$, houve 
um alto índice de uso de gastrostomia (50,67\%) provavelmente por ser um dos procedimentos adotados como alternativa para o uso de sondas de curto prazo em pacientes que necessitem de via alternativa por um tempo maior ${ }^{9}$ e por apresentar uma menor frequência de deslocamento ${ }^{11}$. Em comparação, outro estudo, cujo perfil era de pacientes adultos, foi observado o predomínio da utilização de sonda nasoenteral $(82,53 \%)^{12}$.

\subsection{Sondas utilizadas X Unidades de internação}

O alto índice de uso de gastrostomias na UI Clínica Pediátrica Geral, UTI Neonatologia, UTI Pediátrica Geral se deu pois esses setores apresentam pacientes com comorbidades como paralesia cerebral, na qual há uma debilidade na deglutição ${ }^{13}$, atresia de esôfago, prematuridade de deglutição, e desnutrição.

Sobre as sondas de curta duração (demais sondas), seus usos foram mais observado na UTI Cardiológica e UI Neurologia por caracterizarem-se como UI's póscirúrgicas, ou seja, o paciente não têm previsão de ficar muitos dias internados. As UI da nefrologia e da infectologia não continham pacientes portadores de sonda enteral. Já em outro estudo sobre o perfil dos pacientes em uso de via alternativa, o uso de gastrostomia também foi a principal via observada, correspondendo a $58,07 \%$ só nas unidades em que havia casos com doenças neurológicas, sendo o rebaixamento do nível de consciência a principal razão clínica para tal uso ${ }^{12}$.

\section{4,3 Perfil de medicamentos utilizados via sonda enteral}

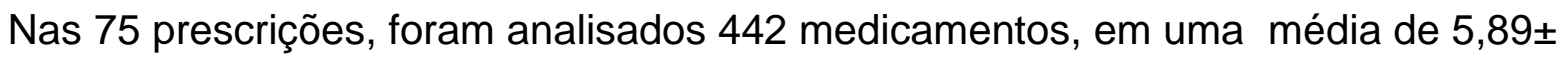
4,30 medicamentos via sonda, por paciente, excluindo medicamentos prescritos por outras vias, como: intravenosa, intramuscular, entre outros. Esse alto índice de medicamentos por paciente, como visto anteriormente, é justificado pelo hospital ser de alta complexicidade. Já em um estudo em um hospital universitário ${ }^{10}$, foi relatada a utilização de $5.6 \pm 2.2$ medicamentos por paciente. Apesar de aparentar ser uma quantidade pequena, é importante ressaltar que quanto maior o número de medicamentos prescritos e administrados ao dia, maior a possibilidade de risco de complicações, como a obstrução das sondas ${ }^{14}$.

Observa-se que o perfil das classes de medicamentos utilizados foram semelhantes 
aos do estudo de ${ }^{10}$ cujos medicamentos mais utilizados também eram analgésicos, sendo o provável motivo de tal utilização o fato de eles promoverem alívio de uma parcela relevante de sintomas, como dor e hipertermia ${ }^{14}$. Além disso, como a maioria dos pacientes internados possuem sua capacidade de se nutrir oralmente fragilizada, é necessário prover essa necessidade através de vitaminas, principalmente a vitamina D3, que são administrados em pacientes até 1 ano de idade, os quais devem ser suplementados diariamente ${ }^{15}$. No estudo realizado, vê-se que isso é disponibilizado para o bem-estar do paciente em questão e, no estudo em comparação, o segundo medicamento mais utilizado foi o ácido fólico (vitamina B9) provavelmente pelo mesmo motivo.

Além disso, pode-se observar no artigo da Silva et al. ${ }^{16}$ que a maior parte das formas farmacêuticas utilizadas foram comprimidos $(62,5 \%)$, formato impróprio ao se considerar a administração via sonda, cujo ideal seria a forma líquida ${ }^{8}$. Em contrapartida, o presente estudo (Figura 1) demonstra um certo avanço, na logística da administração dos medicamentos, ao considerarmos que $35,07 \%$ dos medicamentos são gotas, $7,92 \%$ comprimidos diluídos e $28,28 \%$ líquidos, os quais estão no aspecto mais próximo do ideal. Contudo, tal meta não foi plenamente atingida pelo fato de se utilizar $22,62 \%$ de comprimidos e $6,11 \%$ pó/grânulo, os quais podem causar algum transtorno na hora do manejo, afinal a equipe de enfermagem acaba triturando os comprimidos quando são administrados via sonda, tornando-os fragmentados (em pó). Em seguida, administram-no com outros medicamentos, no formato de pó principalmente. E assim, enfatizando, há possibilidade desses interagirem-se entre si por haver um potencial elevado de interação medicamentosa no fluído entre essas duas formas de apresentação do fármaco ${ }^{17}$. Sendo que esse problema pode ser evitado pelo fato dessa parcela de medicamentos possuírem outras vias disponíveis de administração, por exemplo: no caso do comprimido poderia ser diluído pelo SFH ou, quando disponível, utilizado na apresentação em gotas.

\subsection{Perfil de divergências entre medicamentos prescritos $\mathrm{x}$ realizados}

Das prescrições analisadas, observa-se, como demonstrado na Figura 2 uma quantidade relevante de medicamentos prescritos como comprimido por via oral e realizados por algum tipo de sonda. Lembrando-se que tal divergência, por se tratar de um fármaco sólido, pode ascender as chances de ocorrer inadequações no manuseio das sondas ${ }^{13}$. A exemplo prático, há situações em que a equipe de enfermagem acaba triturando um medicamento a fim desse ser administrado em determinada via (no caso 
desse estudo, as sondas) e, com isso, o revestimento desse fármaco é destruído, alterando a biodisponibilidade do princípio ativo ${ }^{18}$. Consequentemente, altera a resposta terapêutica, ou ainda, aumenta as chances de obstrução do tubo, situações preveníveis que elevam os gastos hospitalares. Logo, ao prescrever a terapêutica, o profissional deve levar em conta a complexidade da forma farmacêutica prescrita, as chances de danos que tal fármaco pode provocar e os custos ${ }^{14}$ para minimizar aqueles erros.

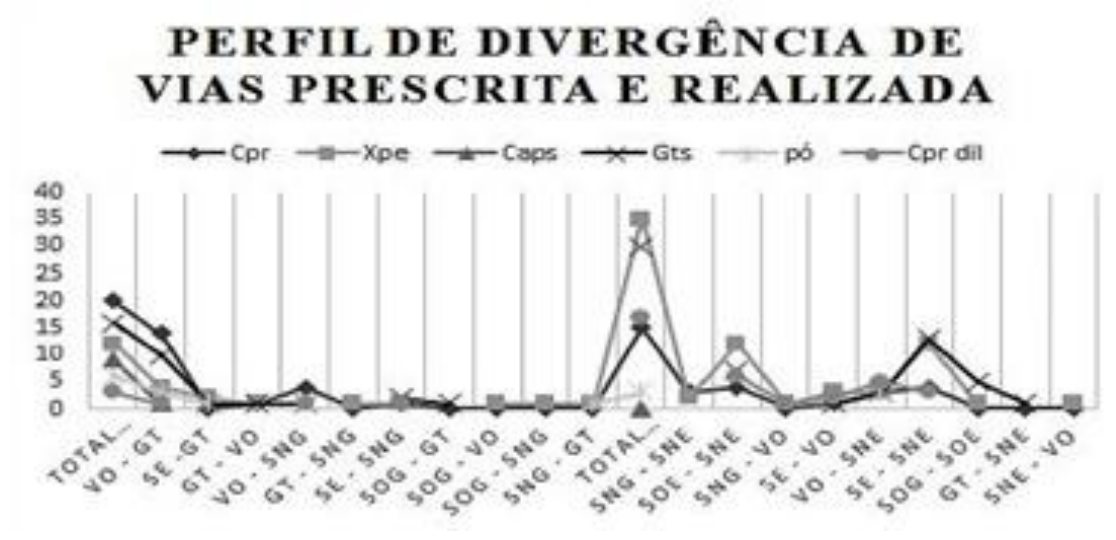

Figura 2. Perfil de divergência de vias prescrita e realizada

Em adição, no presente estudo a sonda em que ocorre maior quantidade de divergência entre prescrição e realização é na sonda enteral (SNE, SOE), correspondente a 53,97\%. Sendo nessa a maior inadequação a administração de líquidos.

Como se observa no presente estudo, a maioria das sondas utilizadas são do tipo gástricas e a divergência predominante, é a inadequação da prescrição como via oral, muitas vezes pelo medicamento não apresentar outra opção de via cadastrada no sistema de prescrição eletrônica, diminuindo a qualidade da assistência oferecida. Afinal, a equipe de enfermagem, responsável pela administração dos medicamentos ${ }^{19}$, necessitará preparar uma nova apresentação do medicamento ao diluir o comprimido, sendo que poderia ser prescrito como diluído Figura 1. Isso sem contar com a maior segurança na dose e no preparo das diluições dos comprimidos, quando realizado pelo SFH. Este processo pode ser relacionado com o modelo do queijo suíço do acidente organizacional de James Reason 20,21 .

\subsection{Flushing}

O flushing é um método de limpeza, o qual deve ser realizado antes e após a 
administração de um medicamento e/ou alimento6,22. Assim, é importante realizar tal procedimento para evitar a obstrução das sondas, por causa dos resíduos dietéticos e medicamentosos, que podem interagir entre si, resultando em um efeito terapêutico ineficaz ${ }^{3}$. Se compararmos esses valores com o estudo de Phillips ${ }^{23}$, em que foi relatado a realização do flushing antes e depois da administração de medicamentos apenas em 12\% dos casos e, na maioria, o flushing foi realizado apenas depois da administração dos medicamentos $\left(96 \%\right.$ dos casos do estudo) ${ }^{23}$. Inicialmente a sensação é de completa seguridade em relação à assistência oferecida, porém, como se observa, mesmo sabendo que o ideal é a realização do flushing antes e depois da administração de medicamentos via sonda, quase em metade da mostra coletada é realizado apenas após, o que pode propiciar a obstrução da sonda e/ou redução do efeito terapêutico no tratamento ${ }^{3}$.

Conclui-se que o perfil dos medicamentos utilizados em pediatria ainda apresenta muitas formas farmacêuticas inapropriadas para a utilização via sonda enteral, necessitando que a equipe de enfermagem realize uma nova preparação a qual pode estar longe do ideal. Portanto, um seguimento farmacoterapêutico mais detalhado é vital para garantir uma terapia eficaz e segura, pois os erros de administração podem levar ao aparecimento de efeitos adversos, debilitando ainda mais a saúde do paciente e, consequentemente, podendo prolongar o seu tempo de internamento.

\section{AGRADECIMENTOS}

Agradecemos à Fundação Araucária pela bolsa auxílio de iniciação científica para as acadêmicas de medicina.

\section{REFERÊNCIAS}

Beckwith MC, Feddema SS, Barton RG, et al. A Guide to Drug Therapy in Patients with Enteral Feeding Tubes: Dosage Form Selection and Administration Methods. Hosp Pharm. 2004;39(3): 225-37.

Blumenstein I, Shastri Y, Stein J. Gastroenteric tube feeding: Techniques, problems and solutions. World Journal of Gastroenterology [Internet]. jul 2014 [acesso em: 29.08.2015];20(26): [8505-24]. Disponível em: < http://www.ncbi.nlm.nih.gov/pmc/articles/ PMC4093701/\#B49 > 
Boullata J. Drug Administration through a Feeding Tube. The Oley Foundation [Internet]. Set-Out 2011 [acesso em: 29.08.2015]. Disponível em: http://www.oley.org/lifeline/Drug_ Administration_Feeding_Tube.html

da Silva MJS, Cava CEM, Pedroso PK, et al. Evaluation of the profile of drug therapy administered through enteral feeding tube in a general hospital in Rio de Janeiro. Brazilian Journal of Pharmaceutical Sciences. 2011, 47(2): 331-7

da Silva, EV. Crianças e medicamentos: os riscos que podem sobrepor os benefícios. Boletim Farmacoterapêutica 2007;1-6.

Didonet J, Predebon S, Schwarzbold CV. Estruturação de orientação farmacêutica para com medicamentos por sonda nasoenteral: um estudo de caso.Rev. Bras. de Farmácia 2011;92(4): 378-83.

Gabriela V, Candido MF, Jesus RP De, et al. PROFILE OF THE USE OF DRUGS ADMINISTERED VIA ENTERAL FEEDING TUBE IN A UNIVERSITY HOSPITAL. Rev. Chil. Nutr. v.37 n.3 Santiago sep. 2010

Goldman L, Ausiello D. Cecil medicina interna. $22^{\text {nd }}$ ed. Rio de Janeiro Elsevier; 2005. P. 1529-33

Gorzoni ML, Della Torre A, Pires SL. Medicamentos e sondas de nutrição. Rev Assoc Med Bras. 2010;56(1):17-21.

Heineck I, Bueno D, Heydrich J. Study on the use of drugs in patients with enteral feeding tubes. Pharm WorldSci 2009; 31(2).P. 145-8.

Izco N, Creus N, Massó J, Codina C, Ribas J. Incompatibilidades fármaco-nutrición enteral: Recomendaciones generales para su prevención. Farm Hosp. 2001;25(1):13-24.

Martini FH, Timmons MJ, Tallitsch RB. Anatomia humana. 6nded.São Paulo: Artmed; 2009. Miranda S. In Costa, MMB, Castro LP. Tópicos em Deglutição e Disfagia. Disfagias Neurológicas da Infância. Rio de Janeiro. MEDSI; 2003, P. 225-31. 
Mota MLS, Barbosa IV, Studart RMB, et al. Avaliação do conhecimento do enfermeiro de unidade de terapia intensiva sobre administração de medicamentos por sonda nasogástrica e nasoenteral. Rev.Latino-Am. Enfermagem [Internet]. set-out 2010 [acesso em: 29.08.2015]; 18(5): [08 telas]. Disponível em: http://www.scielo.br/pdf/rlae/v18n5/pt_08.ppt_08.pdf

Nogueira SCJ, Carvalho APC De, Melo CB De et al. Perfil de pacientes em uso de via alternativa de alimentação internados em um hospital geral. Rev CEFAC. 2012; 15(ahead): 0-0

Pérez-Guisado J. Las dietas cetogénicas: fundamentos y eficacia para la pérdida de peso. Arch Latinoam Nutr. 2008;58(2): 126-31.

Phillips NM, Endacott R. Medication administration via enteral tubes: a survey of nurses' practices.Journal Of Advanced Nursing, Oxford; 2011 v. 67, n. 12, P. 2586-92.

Pickering K. In: WHITE, R; BRADMAN, V. Handbook of Drug administration via Enteral Feeding Tubes. Flushing Enteral Feeding Tubes. Londres: Reino Unido: Pharmaceutical Press, 2007. Cap. 3, p. 11

Reis AMM, Marques TC, Opitz SP, et al. Errors in medicine administration - Profile of medicines: Knowing and preventing. ACTA Paul Enferm. 2010; 23 (2): 181-6.

Reis CT, Martins M, Laguardia J. A segurança do paciente como dimensão da qualidade do cuidado de saúde - um olhar sobre a literatura. Ciência \& Saúde Coletiva 2013; 18(7): 2029-36

Secoli SR. Interações medicamentosas: fundamentos para a prática clínica de enfermagem. Rev da Esc Enferm da USP. 2001;35(1): 28-34.

Spolidoro JVN. In: SOBOTKA, Lubos. Bases da Nutrição Clínica. Nutrição enteral. Rio de Janeiro; 2008. P. 184-85

Wachter RM. Compreendo a segurança do paciente. $2^{\text {nd }}$ ed. Porto Alegre; 2013. P. 21-31

Williams NT. Medication administration through enteral feeding tubes. American Society Of Health-system Pharmacists., Maryland. 2008. v. 65, n. 24, P. 2347-57 